\title{
Kommentar zum Beitrag „Numerische Simulation des Ausbreitungsverhaltens von Wärmeträgerfluid-Inhaltsstoffen aus Erdwärmesonden in Trinkwassereinzugsgebieten" in Grundwasser 25 (3), 189-204 (2020)
}

\author{
Sven Rumohr ${ }^{1}$ \\ Eingegangen: 12. Oktober 2020 / Überarbeitet: 29. Oktober 2020 / Angenommen: 10. Dezember 2020 / Online publiziert: 21. Dezember 2020 \\ (c) Der/die Autor(en) 2020
}

Struß et al. (2020) präsentieren in Ihrem Beitrag die Ergebnisse mittels Modellszenarien durchgeführter Untersuchungen zum Ausbreitungsverhalten von WärmeträgerfluidInhaltsstoffen aus Erdwärmesonden im Zustrombereich von Brunnen in einem Porengrundwasserleiter. Ausgehend von diesen Untersuchungen kritisieren die Autoren, dass sich die Anforderungen der Bundesländer an den Mindestabstand von Erdwärmesonden zu Trinkwasserbrunnen unterscheiden. Die Mindestabstände beurteilen Sie dabei als ,pauschal und naturwissenschaftlich nicht immer begründbar“. Sie sehen daher einen diesbezüglichen Bedarf einer ,generellen nationalen Diskussion“.

Während ich den Wunsch der Autoren nach nachvollziehbaren Regelungen verstehe, muss ich ihre grundsätzliche Kritik fast wortwörtlich zurückweisen: sie ist pauschal und nicht ausreichend begründet. Zwar zeigen die Autoren einige Abstandsregelungen der Bundesländer und die somit bestehenden Unterschiede auf, sie gehen aber nicht näher auf die Hintergründe der einzelnen Regelungen ein.

Der Beitrag geht nicht auf die sich aus dem Wasserhaushaltsgesetz ergebenden Anforderungen an den vorsorgenden Grundwasserschutz und das Handeln der Wasserbehörden ein (Stichwort Besorgnisgrundsatz). Und er geht zu wenig darauf ein, welche Ver- und Gebote in Wasserschutzgebieten z. B. in Bezug auf Bohrungen bestehen, und unter welcher Voraussetzung eine Wasserbehörde überhaupt eine Ausnahmegenehmigung erteilen darf.

Anforderungen an Erdwärmesonden wie z.B. zum Abstand zu einer Gewinnungsanlage werden nicht nur auf-

Sven Rumohr

Sven.Rumohr@hlnug.hessen.de

1 Hessisches Landesamt für Naturschutz, Umwelt und Geologie, Rheingaustraße 186, 65203 Wiesbaden, Deutschland grund eines wassergefährdenden Wärmeträgermittels gestellt. Meines Erachtens wird von den meisten Wasserbehörden die Schaffung von Eintragspfaden in das Grundwasser oder die Herstellung dauerhafter Stockwerksverbindungen durch die für Erdwärmesonden erforderlichen Bohrungen als größeres Risiko gesehen, als die Gefahr einer Leckage (vgl. Ad-Hoc-Arbeitsgruppe Geologie 2011). Eine Diskussion zu Abstandsregelungen kann daher nicht allein anhand des Transportverhaltens von Wärmeträgermitteln im Grundwasser geführt werden.

Letztlich muss auch hinterfragt werden, ob pauschale Ansätze, naturwissenschaftlich begründbar oder nicht, tatsächlich ein Problem darstellen oder sie nicht viel mehr der Vereinfachung und Beschleunigung von Verfahren dienen.

Erfüllt die Errichtung einer Erdwärmesonde in einer Wasserschutzgebietszone einen Verbotstatbestand, kann die zuständige Wasserbehörde unter bestimmten Voraussetzungen eine Ausnahmegenehmigung erteilen. Ein Mindestabstand kann hierbei eine der möglichen Voraussetzungen für eine Ausnahmegenehmigung von einem Verbot sein.

Antragsteller, die bereits in der Planungsphase wissen, dass sie z.B. einen Mindestabstand einhalten können, sind der Ausnahmegenehmigung für ihr Vorhaben einen Schritt näher. Mindestabstände sollten daher eher als Hilfe angesehen und somit positiv gewertet und keinesfalls als einschränkend wahrgenommen werden (Rumohr 2017).

Und selbstverständlich kann eine Ausnahmegenehmigung auch dann beantragt werden, wenn eine publizierte Anforderung nicht eingehalten wird. Die Behörde wird in diesem Fall eine objektive, Fall-bezogene Prüfung des Vorhabens durchführen.

Gerne beteilige ich mich an der weiteren Diskussion zu diesem Thema. Ich erhoffe mir hierzu von den Autoren eine Konkretisierung, wie aus Ihrer Sicht belastbare Mindestabstände in Fällen ermittelt werden können, in denen die Datenlage zur geologischen und geohydraulischen $\mathrm{Si}-$ 
tuation weniger umfassend ist, wie in ihrem bisher eingesetzten Prinzipmodell. Denn Wasserschutzschutzgebiete, in denen es außer der Gewinnungsanlage nur sehr wenige oder überhaupt keine weiteren Messstellen und Aufschlüsse gibt, sind Realität.

Funding Open Access funding enabled and organized by Projekt DEAL.

Open Access Dieser Artikel wird unter der Creative Commons Namensnennung 4.0 International Lizenz veröffentlicht, welche die Nutzung, Vervielfältigung, Bearbeitung, Verbreitung und Wiedergabe in jeglichem Medium und Format erlaubt, sofern Sie den/die ursprünglichen Autor(en) und die Quelle ordnungsgemäß nennen, einen Link zur Creative Commons Lizenz beifügen und angeben, ob Änderungen vorgenommen wurden.

Die in diesem Artikel enthaltenen Bilder und sonstiges Drittmaterial unterliegen ebenfalls der genannten Creative Commons Lizenz, sofern sich aus der Abbildungslegende nichts anderes ergibt. Sofern das betreffende Material nicht unter der genannten Creative Commons Lizenz steht und die betreffende Handlung nicht nach gesetzlichen Vorschrif- ten erlaubt ist, ist für die oben aufgeführten Weiterverwendungen des Materials die Einwilligung des jeweiligen Rechteinhabers einzuholen.

Weitere Details zur Lizenz entnehmen Sie bitte der Lizenzinformation auf http://creativecommons.org/licenses/by/4.0/deed.de.

\section{Literatur}

Ad-Hoc-Arbeitsgruppe Geologie: Fachbericht zu bisher bekannten Auswirkungen geothermischer Vorhaben in den Bundesländern (2011)

Rumohr, S.: Anlagendimensionierung zur Unterschreitung des in Hessen für EWS geforderten Mindestabstandes zur Grundstücksgrenze. bbr 5, 58-68 (2017)

Struß, J., Schäfer, D., Dahmke, A., Köber, R.: Numerische Simulationen des Ausbreitungsverhaltens von Wärmeträgerfluid-Inhaltsstoffen aus Erdwärmesonden in Trinkwassereinzugsgebieten. Grundwasser 25(3), 189-204 (2020)

Hinweis des Verlags Der Verlag bleibt in Hinblick auf geografische Zuordnungen und Gebietsbezeichnungen in veröffentlichten Karten und Institutsadressen neutral. 